\title{
Fabry disease: a mimic for obstructive hypertrophic cardiomyopathy?
}

\author{
S R Ommen, R A Nishimura, W D Edwards
}

Heart 2003;89:929-930

$\mathrm{T}$ he evaluation of hypertrophic cardiomyopathy (HCM) involves the careful consideration of diseases that can mimic its appearance, such as aortic stenosis, poorly controlled hypertension, and infiltrative processes such as cardiac amyloidosis. Fabry disease, an inherited deficiency of the enzyme $\alpha$ galactosidase A, in particular, has recently received much attention for new and nearly curative treatments. ${ }^{12}$ Exogenous administration of the deficient enzyme has been shown to clear microvascular endothelial deposits, which are integral to disease manifestation, in the kidney, skin, and heart. Interestingly, isolated cardiac Fabry disease may exist without other overt manifestations. In one study, 3\% of male subjects with myocardial hypertrophy were found to harbour this enzyme deficiency, ${ }^{3}$ whereas another study showed that approximately $4 \%$ of patients referred to a tertiary centre for evaluation of HCM actually had evidence of Fabry disease. ${ }^{4}$ If this storage disease were misdiagnosed as HCM, patients who could be treated effectively with medication might be subjected to aggressive procedures such as septal ablation or surgical septal myectomy. We sought to determine the frequency of Fabry disease among patients who underwent surgical myectomy for the relief of symptomatic obstructive HCM.

\section{PATIENTS AND METHODS}

This investigation was approved by the Mayo Foundation institutional review board. Medical histories were reviewed from 100 consecutive patients who had undergone subaortic ventricular septal myectomy for the treatment of HCM at the Mayo Clinic from January 1997 through December 1999. Patients with hypertension or valvar disease were not included. Patient demographics, clinical status, echocardiographic data, and haemodynamic data were recorded. The myectomy tissue was examined with a Philips CM12/STEM transmission electron microscope for evidence of Fabry disease. By transmission electron microscopy, the presence of myeloid figures within the majority of myocytes is characteristic of symptomatic Fabry cardiomyopathy. Conversely, their absence from all myocytes examined by transmission electron microscopy is indicative of the absence of Fabry cardiomyopathy.

\section{RESULTS}

The 100 patients had a mean (SD) age of 51 (16) years at the time of myectomy and 45 (17) years at the time of diagnosis; 44 were men. The average maximal left ventricular septal wall thickness was 21 (6) $\mathrm{mm}$, and the posterior wall was 15 (5) $\mathrm{mm}$. All patients had severely limiting angina, dyspnoea, or exertional pre-syncope, despite pharmacological treatment. The resting left ventricular outflow tract gradient was 77 (46) $\mathrm{mm} \mathrm{Hg}$ preoperatively and 4 (5) $\mathrm{mm} \mathrm{Hg}$ postoperatively. Electron microscopy examination of the 100 myectomy specimens revealed no myeloid figures characteristic of Fabry disease.

\section{DISCUSSION}

HCM and Fabry disease are distinct inherited disorders that can lead to myocardial hypertrophy. The initial treatment in obstructive HCM includes pharmacologic therapy. If symptoms cannot be controlled with pharmacotherapy, then patients have been offered dual chamber pacing, surgical septal myectomy, or catheter based septal ablation.

Isolated cardiac Fabry disease has been reported in 3-5\% of patients with undifferentiated myocardial hypertrophy and in a similar proportion of patients referred for tertiary evaluation of HCM. ${ }^{34}$ Recently, Fabry disease has been shown to respond to enzyme replacement therapy, including an improvement in cardiac function and regression of hypertrophy. ${ }^{5}$

The implications of an incorrect diagnosis are considerable. If Fabry disease is misdiagnosed as HCM, patients could be denied treatment with enzyme replacement and exposed to the invasive treatment strategies for obstructive HCM, which have procedural mortality rates of $1-3 \%$. Fortunately, none of the patients in the current study had evidence of a lysosomal storage disorder.

Our findings contrast those of Sachdev and colleagues ${ }^{4}$ and may be related to the specific morphologic features and pattern of hypertrophy. The major difference between our patients and the previous analyses is that all of our patients had asymmetrical hypertrophy causing severe outflow obstruction, whereas five of six patients with Fabry disease had concentric hypertrophy in the study by Sachdev and colleagues. ${ }^{4}$ Classically, cardiac manifestations of Fabry disease have involved a more generalised, diffuse myocardial wall thickening. The obstructive form of HCM, occurring in $25-30 \%$ of patients with HCM, often has hypertrophy isolated to the basal septum. It is these patients who are represented in our analysis and are the optimal candidates for surgical myectomy or alcohol ablative techniques.

Our patients included both sexes, in contrast to the previous studies. Fabry disease is an $\mathrm{X}$ linked recessive disease; however, up to a fourth of female carriers have been reported to have left ventricular hypertrophy. It has been suggested that Fabry disease is more likely in late onset hypertrophy. Our patients had a mean age of 51 years, and two thirds were older than 40 years.

Although a thorough investigation for reversible causes of left ventricular hypertrophy remains important, this investigation of 100 consecutive patients suggests that among patients with severely symptomatic, obstructive, asymmetrical basal septal hypertrophy, Fabry disease is unlikely.

\section{Authors' affiliations}

S R Ommen, R A Nishimura, Division of Cardiovascular Diseases and Internal Medicine, Mayo Clinic, Rochester, Minnesota, USA W D Edwards, Division of Anatomic Pathology, Mayo Clinic

C) Copyright 2003 Mayo Foundation 
Correspondence to: Steve R Ommen, MD, Division of Cardiovascular Medicine and Internal Medicine, Mayo Clinic, 200 First Street SW, Rochester, MN 55905, USA; ommen.steve@mayo.edu

Accepted 10 March 2003

\section{REFERENCES}

1 Schiffmann R, Kopp JB, Austin HA III, et al. Enzyme replacement therapy in Fabry disease: a randomized controlled trial. JAMA $2001 ; 285: 2743-9$
2 Pastores GM, Thadhani R. Enzyme-replacement therapy for Anderson-Fabry disease. Lancet 2001;358:601-3.

3 Nakao S, Takenaka T, Maeda M, et al. An atypical variant of Fabry's disease in men with left ventricular hypertrophy. N Engl J Med $1995 \cdot 333: 288-93$

4 Sachdev B, Takenaka T, Teraguchi $\mathrm{H}$, et al. Prevalence of

Anderson-Fabry disease in male patients with late onset hypertrophic cardiomyopathy. Circulation 2002;105:1407-11

5 Frustaci A, Chimenti C, Ricci R, et al. Improvement in cardiac function in the cardiac variant of Fabry's disease with galactose-infusion therapy. N Engl J Med 2001;345:25-32.

\section{IMAGES IN CARDIOLOGY}

\section{Improved myocardial viability following intramyocardial autologous bone marrow injection after acute myocardial infarction}

$A^{s}$ 57 year old man was admitted to our hospital with acute anterolateral myocardial infarction. He was treated with intravenous thrombolysis within 120 minutes after the initial chest pain. Creatine phosphokinase peaked at $1890 \mathrm{U} / \mathrm{l}$ after six hours. Coronary angiography obtained five days postinfarction demonstrated one vessel left anterior descending artery (LAD) disease not requiring balloon angioplasty. Echocardiography revealed apical and mid anterolateral hypo- to akinesia that was in accordance with an anterolateral defect in the Tc99m-MIBI rest technique. Six weeks post-infarction $600 \mathrm{ml}$ of bone marrow were harvested from the patient's hipbone. NOGA non-fluoroscopic electromechanical mapping (Biosense Webster) at this time showed significantly reduced voltage values in the anterior, anterolateral and septal regions, indicated by the red colour (panel A). A total of $2 \times 10^{9}$ bone marrow mononuclear cells $(5.2 \mathrm{ml})$ were implanted at 12 sites into the transition zone between scar and viable myocardium by NOGA guided injection.

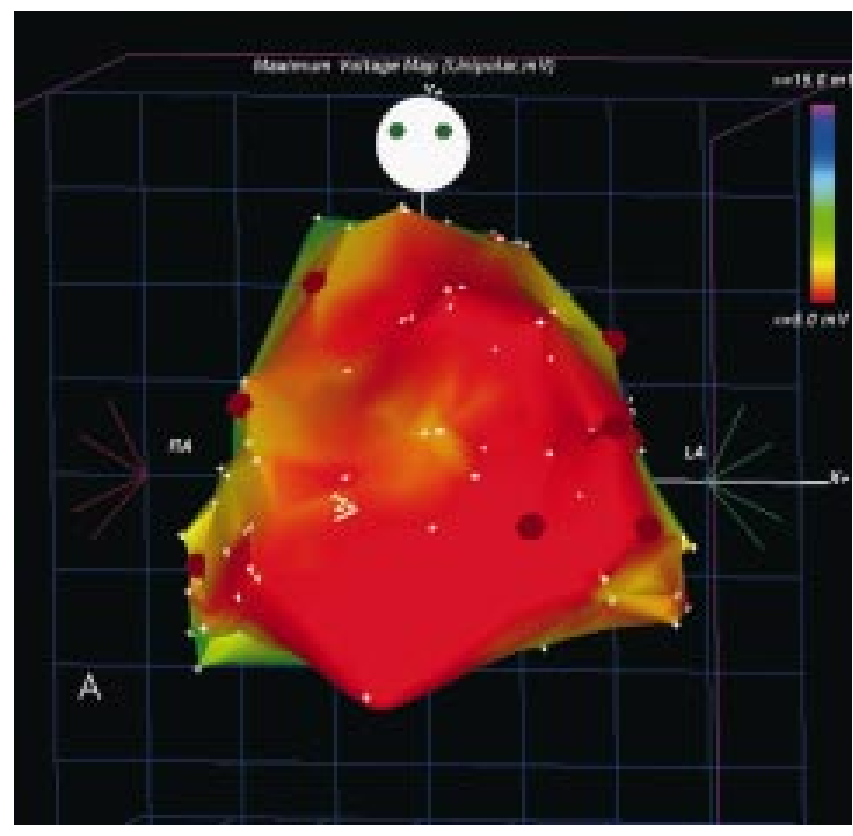

In the six month follow up NOGA, the anteroseptal and anterolateral region was green-yellow, suggesting improved viability in these areas (panel B). Myocardial perfusion and viability imaging using the stress and glyceryl trinitrate enhanced Tc99m-MIBI rest technique showed a decrease in resting defect size from $34 \%$ to $25 \%$. LAD territorial extentthat is, uptake extent in the area-of-risk-had decreased from $65 \%$ to $46 \%$. Left ventricular ejection fraction obtained by gated single photon emission computed tomography had improved from $33 \%$ to $41 \%$.

The data demonstrate improved viability and perfusion six months after anterior myocardial infarction and percutaneous intramyocardial autologous bone marrow transplantation.

G Beran

D Glogar

I M Lang

berangilbert@yahoo.com

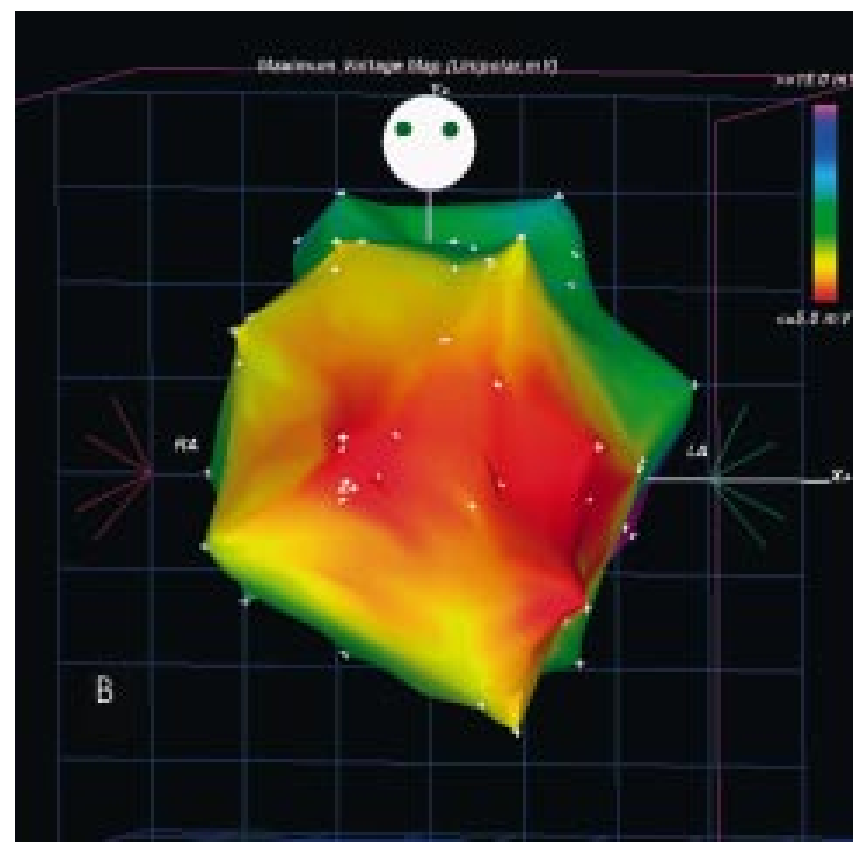

NOGA baseline unipolar voltage map before (panel A) and six months after bone marrow transplantation (panel B). Red areas represent electrical activity below $5 \mathrm{mV}$, indicating low viability (colour bar ranging from red $<5 \mathrm{mV}$ to purple $>15 \mathrm{mV}$ ). Brown dots mark the intramyocardial injection sites. 